\title{
Finite-time synchronization of fractional-order simplest two-component chaotic oscillators
}

\author{
Romanic Kengne ${ }^{1,2}$, Robert Tchitnga ${ }^{1,2}$, Anicet Mezatio ${ }^{1,2}$, Anaclet Fomethe ${ }^{3}$, and Grzegorz Litak An, a $^{4,5}$ \\ 1 Research Group on Experimental and Applied Physics for Sustainable Development, Faculty of Science, \\ Department of Physics, University of Dschang, P.O. Box 412, Dschang, Cameroon \\ 2 Laboratory of Electronics and Signal Processing Faculty of Science, Department of Physics University of Dschang, \\ P.O. Box 67, Dschang, Cameroon \\ 3 Laboratoire de Mécanique et de Modélisation des Systèmes, L2MS, Department of Physics, Faculty of Science, University of \\ Dschang, P.O. Box 67, Dschang, Cameroon \\ 4 Lublin University of Technology, Faculty of Mechanical Engineering, Nadbystrzycka 36, 20-618 Lublin, Poland \\ 5 AGH University of Science and Technology, Faculty of Mechanical Engineering and Robotics, Department of Process \\ Control, Mickiewicza 30, 30-059 Krakow, Poland
}

Received 7 August 2016 / Received in final form 27 February 2017

Published online 10 May 2017

(C) The Author(s) 2017. This article is published with open access at Springerlink.com

\begin{abstract}
The problem of finite-time synchronization of fractional-order simplest two-component chaotic oscillators operating at high frequency and application to digital cryptography is addressed. After the investigation of numerical chaotic behavior in the system, an adaptive feedback controller is designed to achieve the finite-time synchronization of two oscillators, based on the Lyapunov function. This controller could find application in many other fractional-order chaotic circuits. Applying synchronized fractionalorder systems in digital cryptography, a well secured key system is obtained. Numerical simulations are given to illustrate and verify the analytic results.
\end{abstract}

\section{Introduction}

Over decades, the study of fractional-order systems is gaining ground both in theory and practice. It has been found that in many practical cases, systems can be more adequately described using fractional-order differential equations [1-11]. Furthermore, fractional-order derivatives are excellent instruments for the description of memory and hereditary properties of various materials and processes [12]. Fractional-order systems have also the advantage of possessing higher degrees of freedom compared to their integer-order counterparts [13].

Recently, increasing interest has been devoted to the study of chaotic phenomena in fractional-order counterparts of integer-order dynamical systems [14-23]. Surprisingly almost all of the existing fractional-order chaotic systems found so far in the literature shared a common characteristic: there are operating in low frequency range which is somehow a drawback for their potential applications in secure communication and control processing.

Among various possible applications of chaos, synchronization of chaotic systems in secure communication becomes inescapable [24,25]. Yet, several authors are also interested in the synchronization of the fractional-order

a e-mail: g.litak@pollub.pl chaotic systems $[22,23,26-35]$. The transient time preceding the synchronization state of chaotic oscillators is crucial as it unfortunately corresponds to the laps of time during which the encoded message cannot be recovered or sent. More than a difficulty, this is a catastrophe in digital telecommunications since the first bits of standardized bit strings always contain signalization data, i.e, the identity card of the message. Hence, it clearly appears that the synchronization time has to be known and minimized, so to make the synchronization be achieved as fast as possible. In this context, there is a relentless activity in the study of finite-time chaos synchronization $[36,37]$ and gradually of fractional-order systems [38-40]. Unfortunately, in many of these references dealing with synchronization, for example in the previous three, applications in secure communication are missing. The authors of reference [22], although working on secure communication, use rather an infinitetime synchronization technique applied on a low frequency operating circuit.

Recent examples of two-component electronic circuits [41] operating at high frequency $[42,43]$ have been reported. In spite of the simplicity of [43] made solely of a transistor and a tapped coil, Joana et al. [44] have revealed the presence of infinite families of spiral phases of stability in its phase diagrams. Meanwhile Nguimdo et al. [45] coupling three such circuits in a unidirectional ring 
studied with success their potential applications to random bit generator. That circuit appears to be a good candidate to tackle some of the problems noted in some of the above references as drawbacks. The idea of finite-time synchronization of fractional-order has recently been tackled in reference [46]. Based on a linear feedback controller the authors presented for the first time the finite-time inputto-state stable theory of fractional-order dynamical systems in the goal to achieve complete synchronization in the presence of uncertainties and disturbance. Numerical simulations were given to support the feasibility of the presented synchronization scheme. Our approach can be considered as another aspect of similar development.

In the present paper, based on the Lyapunov function a fractional adaptive finite-time synchronization reflecting the method in [47] is proposed for fractional-order versions of the circuit in [43]. Then, a new key system for encryption on digital cryptography is found, using the proposed finite-time synchronization scheme which is more secure and more powerful than an existing key system of integerorder synchronization [22].

High frequency signals are characterized among others by a brighter bandwidth, compared to low frequency ones. In chaos-based communications, the speed of the message which can be well concealed within a chaotic jamming depends on the bandwidth of the chaotic signal. If the bandwidth is large, then the speed of the message will be significant, leading to high processing speeds [45]. For this purpose, a great interest is placed on chaotic circuits operating at high frequency. Hence the importance of the synchronization of chaotic systems operating at such frequencies with respect to the synchronization of their counterparts operating at low frequencies.

The outline of this paper foresees in Section 2 the presentation of the fractional-order mathematical model derived from the corresponding electronic device as well as a brief investigation of its chaotic dynamics. Section 3 deals with the adaptive finite-time synchronization of two identical fractional-order two-component oscillators. Here, the active controller is designed and the theoretical settling time is developed. Numerical simulations are performed to show the effectiveness of the synchronization scheme. In Section 4, using the results of the finite-time fractionalorder synchronization, a digital cryptography is investigated. In that same section, the Fibonacci $Q$ matrix and the new key using the frequency of the fractional-chaotic circuit help to secure a digital message. Finally, in the last section, conclusions are provided.

\section{Dynamic of fractional-order simplest two-component oscillator}

\subsection{Circuit model and mathematical modeling}

A fractional order dynamical system is an nontrivial extension with respect to integer one. The main difference would focus on in the memory of system. This memory modifies effective dimensionality of the dynamical system.

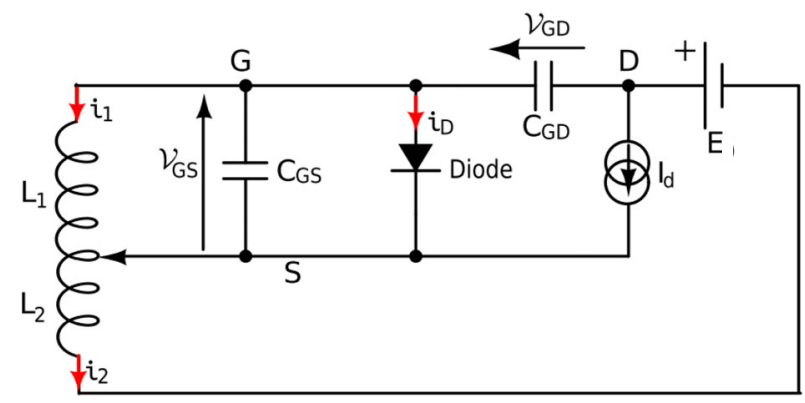

Fig. 1. Equivalent circuit of the simplest two-component oscillator proposed in [43], using the simplified Giacoletto dynamic model of the JFET for small signals at high frequency and governed by the fractional-order four-dimensional flow in equation (1).

Figure 1 represents the high-frequency small-signal equivalent model of the two-component oscillator modeled by Tchitnga et al. in [43]. In that model the currents flowing through the parasitic capacitors $C_{G S}$ and $C_{G D}$ depend on the integer-order derivative of the voltage across the same parasitics capacitors respectively; while the voltages across the two parts $L_{1}$ and $L_{2}$ of the inductor depend on the integer-order derivative of currents flowing through those two inductor parts. The internal resistance of the tapped coil is neglected.

In the present paper we consider that the currents flowing through the parasitic capacitors $C_{G S}$ and $C_{G D}$ depend rather on the fractional-order derivative of the corresponding voltages across these capacitors. Furthermore, the voltages across the two parts $L_{1}$ and $L_{2}$ of the inductor will now depend on the fractional-order derivative of currents flowing them, while the internal resistance of the tapped coil remains neglected. To remind, the fractionalorder, $q(0<q<1)$, commensurate circuit is considered and according to the Kirchhoff laws. For simplicity, we assumed the same fractional order in the corresponding equations:

$$
\left\{\begin{array}{l}
\frac{d^{q} V_{G S}}{d t^{q}}=\frac{1}{C_{G S}}\left(-i_{1}+i_{2}-i_{D}-I_{d}\right) \\
\frac{d^{q} V_{G D}}{d t^{q}}=\frac{1}{C_{G D}}\left(-i_{2}+I_{d}\right) \\
\frac{d^{q} i_{1}}{d t^{q}}=\frac{1}{L_{1}} V_{G S} \\
\frac{d^{q} i_{2}}{d t^{q}}=\frac{1}{L_{2}}\left(-V_{G S}+V_{G D}+E\right) .
\end{array}\right.
$$

Here $i_{D}$ and $I_{d}$ represented below are nonlinear currents flowing through the diode and from the drain to the source of the transistor respectively:

$$
i_{D}=I_{S}\left[\exp \left(\frac{V_{G S}}{V_{T}}\right)-1\right]
$$



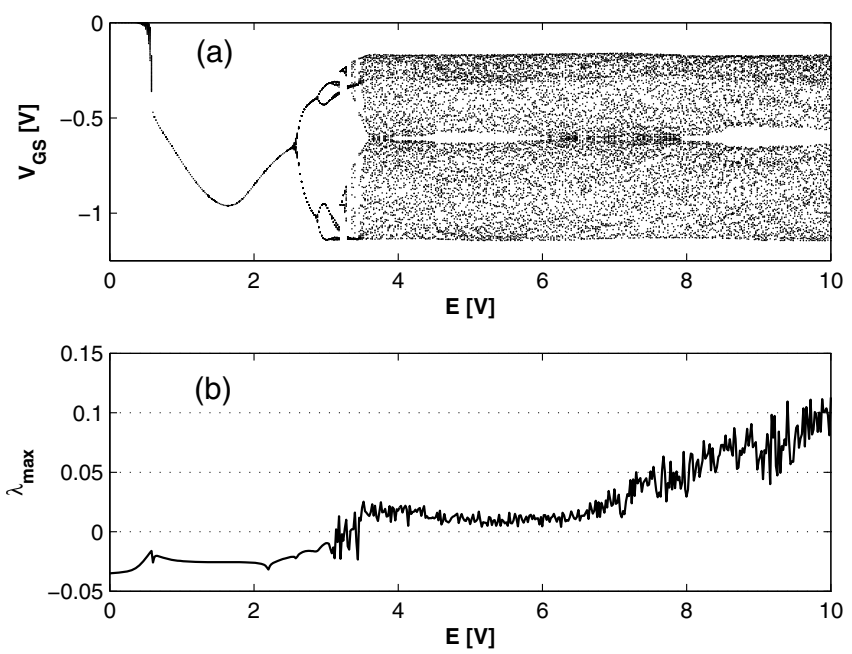

Fig. 2. (a) Bifurcation diagram of the local extrema for the variable $V_{G S}$ and (b) the corresponding Largest Lyapunov exponent for $q=0.96$ while the bias voltage source $E$ increases from 0 to $10 \mathrm{~V}$. The chaotic behavior is observed for the values of control parameters corresponding to the positive Largest Lyapunov exponent.

and

$$
I_{d}=\left\{\begin{array}{rr}
0 \quad \text { if } \quad \mathrm{V}_{\mathrm{GS}} \leq V_{G \text { Soff }} \\
g_{m 0}\left(V_{G S}-V_{G \text { Soff }}\right)^{2} \quad \text { if } \quad \mathrm{V}_{\mathrm{GD}} \leq V_{G S o f f} \\
g_{m 0}\left(V_{G S}-V_{G D}\right)\left(V_{G S}+V_{G D}-2 V_{G \text { Soff }}\right) \\
\text { if } \quad \mathrm{V}_{\mathrm{GD}} \geq V_{G \text { Soff }}
\end{array}\right.
$$

The experimental values of parameters are given as follows: $C_{G S}=3.736 \mathrm{pf}, C_{G D}=3.35 \mathrm{pf}, L_{1}=24.5 \mu \mathrm{H}$, $L_{2}=4 \mu \mathrm{H}, I_{S}=33.57 \mathrm{fA}, V_{G S o f f}=-1.409 \mathrm{~V}, g_{m 0}=$ $1.754 \mathrm{~mA} \mathrm{~V}^{-2}$, and $V_{T}=25 \mathrm{mV}$.

The voltage source $E$ and the commensurate fractional-order $q$ are used as control parameters for the numerical characterization of chaotic phenomena in the circuit. The fractional-order system (1) is solved by means of the improved version of Adam-Bashforth-Moulton numerical algorithm while the Lyapunov exponents have been obtained by means of the Wolf algorithm [48]. Note that this algorithm is used as a rough approximation [49]. It could give wrong estimations of the maximal Lyapunov exponnent account for a variable phase space dimension occuring in systems with memory.

\subsection{Bifurcations}

Figure 2 depicts the bifurcation diagram (Fig. 2a) and the corresponding Lyapunov exponent (Fig. 2b), respectively. The commensurate fractional-order is fixed at $q=0.96$ and the control parameter varies in the range $(0<E<$ 10) V. Period doubling route to chaos occurs in this fractional-order model from $E=0.6 \mathrm{~V}$ to $E=3.18 \mathrm{~V}$, while chaotic behavior is recorded for $(3.52<E<10) \mathrm{V}$, confirmed by the largest Lyapunov exponent.
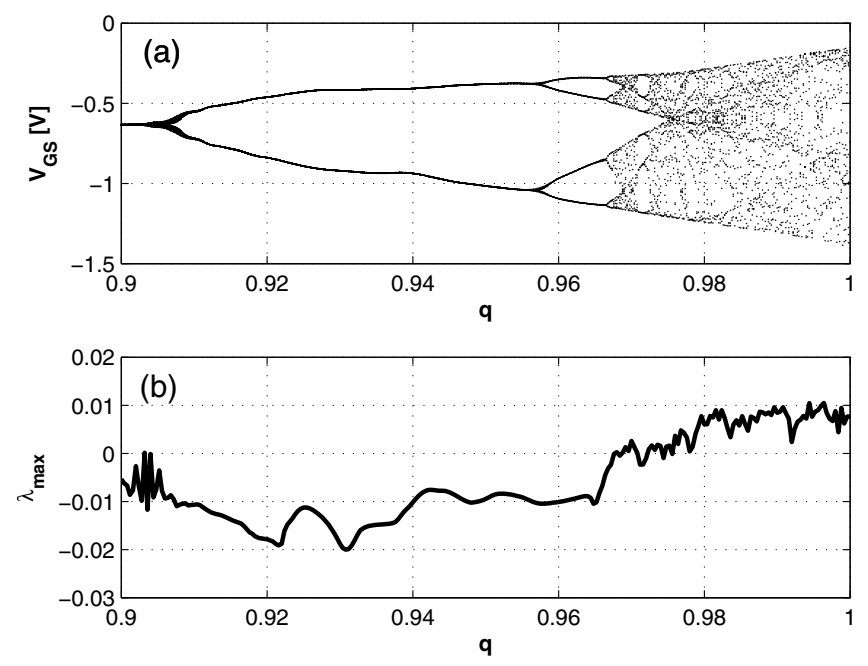

Fig. 3. (a) Bifurcation diagram of the local extremal for the variable $V_{G S}$ plotted when the control parameter $q$ increases from 0.91 to 1 and bias voltage source fixed at $E=3 \mathrm{~V}$; (b) the corresponding Largest Lyapunov exponent for the same range of the control parameter $q$.

Repeating that calculations by keeping the bias voltage source (previous control parameter) fixed at $E=3 \mathrm{~V}$ and varying rather the commensurate fractional-order $0.9<$ $q<1$, Figure 3 is obtained. The new bifurcation diagram (Fig. 3a) and its corresponding largest Lyapunov exponent (Fig. 3b) display other cases with rich dynamics, when the control parameter $q$ varies in the range $0.9<q<1$. Period doubling sequences in the circuit $\left(P_{1} \rightarrow P_{2} \rightarrow\right.$ $\left.P_{4} \rightarrow P_{8}\right)$ can be noted in Figure 3 a when $q$ increases from 0.9 to 0.9674 . Here, the Lyapunov exponent is negative (see Fig. 3b).

A jump to period-six is observed for $0.9708<q<$ 0.9724 before the dynamic behavior in the circuit falls into chaos, materialized by the positive Lyapunov exponents (Fig. 3b).

For the selected couple of parameters $(E=4 \mathrm{~V}, q=$ $0.96)$ in the chaotic domain, the power spectrum with its peak at around $F=0.15 \mathrm{GHz}$ (Fig. 4a) confirms that the fractional-order two-component chaotic circuit oscillates at high frequencies. The Poincaré map (Fig. 4b) plotted in the plan $V_{G D}-i_{2}$ for $V_{G S}=0.6$ and the phase portrait (Fig. 4c) are also given.

Remark 1: The larger range of variation of the bias voltage source (Fig. 3) for chaos in the fractional-order two-component oscillator compared to the range of the same parameter for chaos in its integer-order counterpart plotted in reference [43] is an advantage for the first, in secure communication.

\section{Finite-time adaptive synchronization of fractional-order two-component oscillators}

\subsection{Preliminaries}

In this section, the finite-time adaptive synchronization of two identical simplest two-component oscillators 


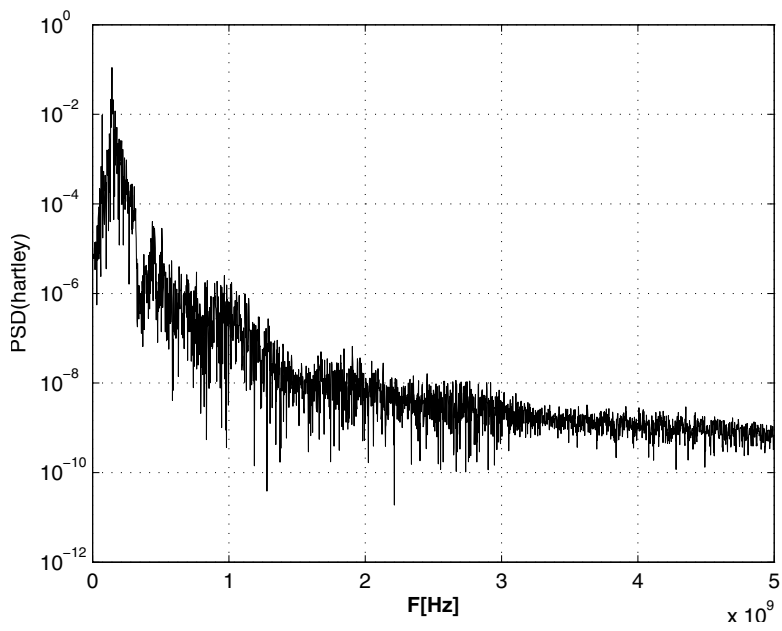

(a)

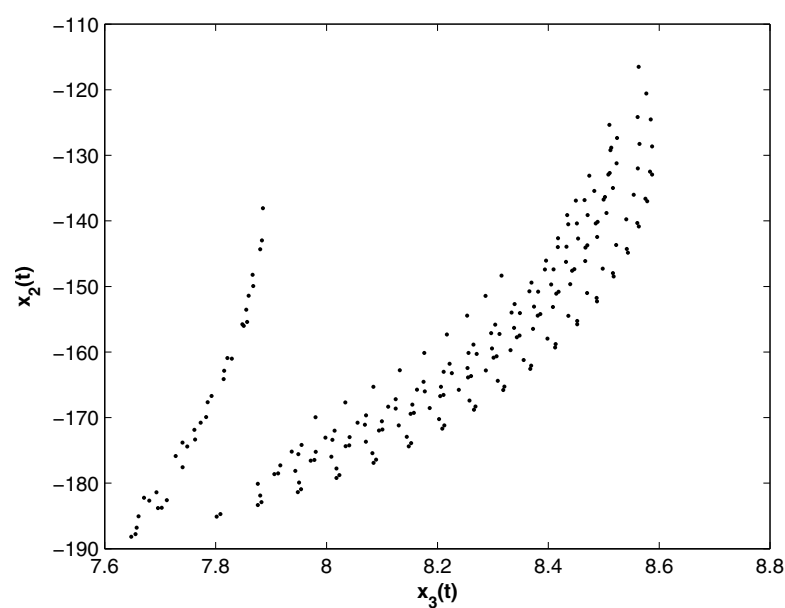

(b)

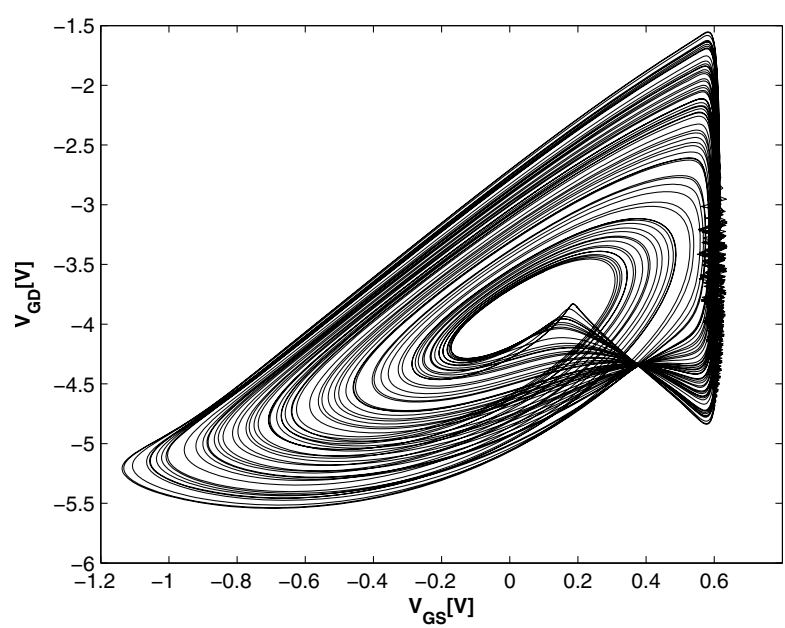

(c)

Fig. 4. Various graphs plotted for the couple of control parameters $(E=4 \mathrm{~V}, q=0.96)$ : (a) power spectrum of the fractionalorder two-component oscillator confirming the high frequency oscillatory nature of the circuit under study; (b) Poincaré map plotted in the $\left(V_{G S}-i_{2}\right)$ plane for $V_{G D}=0.6 \mathrm{~V}$, proving the chaotic behavior of the oscillator; (c) $\left(V_{G S}-V_{G D}\right)$ plane phase portrait for the above values of the parameters. is investigated under consideration of the following master system

$$
\left\{\begin{array}{l}
\frac{d^{q} V_{G S}^{m}}{d t^{q}}=\frac{1}{C_{G S}}\left(-i_{1}^{m}+i_{2}^{m}-i_{D}^{m}-I_{d}^{m}\right) \\
\frac{d^{q} V_{G D}^{m}}{d t^{q}}=\frac{1}{C_{G D}}\left(-i_{2}^{m}+I_{d}^{m}\right) \\
\frac{d^{q} i_{1}^{m}}{d t^{q}}=\frac{1}{L_{1}} V_{G S}^{m} \\
\frac{d^{q} i_{2}^{m}}{d t^{q}}=\frac{1}{L_{2}}\left(-V_{G S}^{m}+V_{G D}^{m}+E\right)
\end{array}\right.
$$

and the corresponding slave system:

$$
\left\{\begin{aligned}
\frac{d^{q} V_{G S}^{s}}{d t^{q}}= & \frac{1}{C_{G S}}\left(-i_{1}^{s}+i_{2}^{s}-i_{D}^{s}-I_{d}^{s}\right. \\
& \left.\quad-\xi \operatorname{sign}\left(V_{G S}^{s}-V_{G S}^{m}\right)-\xi u(t)\right) \\
\frac{d^{q} V_{G D}^{s}}{d t^{q}}= & \frac{1}{C_{G D}}\left(-i_{2}^{s}+I_{d}^{s}\right) \\
\frac{d^{q} i_{1}^{s}}{d t^{q}}= & \frac{1}{L_{1}} V_{G S}^{s} \\
\frac{d^{q} i_{2}^{s}}{d t^{q}}= & \frac{1}{L_{2}}\left(-V_{G S}^{s}+V_{G D}^{s}+E\right) .
\end{aligned}\right.
$$

The term $-\xi \operatorname{sign}\left(V_{G S}^{s}-V_{G S}^{m}\right)-\xi u(t)$ represents the nonlinear controller, where $u(t)$ is the adaptive feedback coupling designed to achieve finite-time synchronization, while the remainder parts contribute to synchronize both master and slave oscillators at an established time as well as to stabilize the response dynamics.

Let the error function be defined as: $e_{1}(t)=V_{G S}^{s}(t)-$ $V_{G S}^{m}(t), e_{2}(t)=V_{G D}^{s}(t)-V_{G D}^{m}(t), e_{3}(t)=i_{1}^{s}(t)-i_{1}^{m}(t)$ and $e_{4}(t)=i_{2}^{s}(t)-i_{2}^{m}(t)$. The error dynamical system resulting from equations (4) and (5) can be written as:

$$
\left\{\begin{aligned}
& \frac{d^{q} e_{1}}{d t^{q}}= \frac{1}{C_{G S}}\left(-e_{3}+e_{4}-\left(i_{D}^{s}-i_{D}^{m}\right)-\left(I_{d}^{s}-I_{d}^{m}\right)\right. \\
&\left.-\xi \operatorname{sign}\left(e_{1}\right)-\xi u(t)\right) \\
& \frac{d^{q} e_{2}}{d t^{q}}= \frac{1}{C_{G D}}\left(-e_{4}+\left(I_{d}^{s}-I_{d}^{m}\right)\right) \\
& \frac{d^{q} e_{3}}{d t^{q}}=\frac{1}{L_{1}} e_{1} \\
& \frac{d^{q} e_{4}}{d t^{q}}=\frac{1}{L_{2}}\left(-e_{1}+e_{2}\right) .
\end{aligned}\right.
$$

Setting $\phi_{1}=\left(i_{D}^{s}-i_{D}^{m}\right)$ and $\phi_{2}=\left(I_{d}^{s}-I_{d}^{m}\right)$, equation (6) becomes:

$$
\left\{\begin{array}{l}
\frac{d^{q} e_{1}}{d t^{q}}=\frac{1}{C_{G S}}\left(-e_{3}+e_{4}-\phi_{1}-\phi_{2}-\xi \operatorname{sign}\left(e_{1}\right)-\xi u(t)\right) \\
\frac{d^{q} e_{2}}{d t^{q}}=\frac{1}{C_{G D}}\left(-e_{4}+\phi_{2}\right) \\
\frac{d^{q} e_{3}}{d t^{q}}=\frac{1}{L_{1}} e_{1} \\
\frac{d^{q} e_{4}}{d t^{q}}=\frac{1}{L_{2}}\left(-e_{1}+e_{2}\right)
\end{array}\right.
$$


The slave system (5) synchronizes with the master system (4) in a finite-time if and only if it exists a final time $t_{r}$ such that $\lim _{t \rightarrow t_{r}}\left\|e_{i}(t)\right\|=0$ with $i=1, \ldots, 4$.

Assumption 1: The nonlinear functions vectors $\phi_{1}\left(e_{1}, e_{2}\right)$, and $\phi_{2}\left(e_{1}, e_{2}\right)$ with $\phi_{1}(0,0)=\phi_{2}(0,0)=0$ respect the Lipschitz condition, if and only if it exists two positive scalars numbers $\chi_{1}$ and $\chi_{2}$ respectively such that: $\left\|\phi_{1}\left(e_{1}, e_{2}\right)\right\| \leq \chi_{1}\left\|e_{1}(t)\right\|$ and $\left\|\phi_{2}\left(e_{1}, e_{2}\right)\right\| \leq \chi_{2}\left\|e_{2}(t)\right\|$.

Assumption 2: Considering two vectors $X$ and $Y$, it exists three positive scalars numbers $\chi_{1}, \chi_{2}$ and $\eta$ such that: $\chi_{1} X X^{T}+\chi_{2} X Y+\chi_{2} Y Y^{T} \leq \eta Y Y^{T}$.

\subsection{Main results}

Theorem 1: For master system (4) and slave system (5), if we choose the adaptive feedback controller $u(t)$ as:

$$
D^{q} u=\frac{\Gamma(2-q)}{\Gamma(2)} u^{q-1}\left(\xi\left|e_{1}\right|+\xi e_{1} u-\eta e_{1}^{2}-\beta\right) \operatorname{sign}(u)
$$

with $\beta$ a positive constant, then the two dynamic systems (4) and (5) will enter into a state of synchronization after a finite-time depending on $\beta$ and according to the relation:

$$
\begin{aligned}
t_{r}^{q} \leq \frac{1}{2 \beta}\left(C_{G S} e_{1}^{2}(0)+C_{G D} e_{2}^{2}(0)\right. & +L_{1} e_{3}^{2}(0) \\
& \left.+L_{2} e_{4}^{2}(0)\right)+\frac{|u(0)|}{\beta} .
\end{aligned}
$$

Proof: Consider the following Lyapunov candidate function:

$$
V=(1 / 2) e W e^{T}+|u(t)|
$$

where $W$ is a symmetric positive definite real matrix, chosen as $W=\left(\begin{array}{cccc}C_{G S} & 0 & 0 & 0 \\ 0 & C_{G D} & 0 & 0 \\ 0 & 0 & L_{1} & 0 \\ 0 & 0 & 0 & L_{2}\end{array}\right)$.

The corresponding $J$ function for this Lyapunov function is:

$$
J=e^{T} W\left(D^{q} e\right)+D^{q}|u(t)| .
$$

Exploiting equations (7) and (11), $J$ can be expanded as:

$$
\begin{aligned}
J= & -\phi_{1} e_{1}-\phi_{2} e_{1}-\xi\left|e_{1}\right|-\xi e_{1} u+\phi_{2} e_{2} \\
& +\frac{\Gamma(2)}{\Gamma(2-q)} u^{1-q} \operatorname{sign}(u) D^{q} u .
\end{aligned}
$$

Using assumption 1, equation (12) can be rewritten as:

$$
\begin{aligned}
J \leq \chi_{1} e_{1}^{2}+\chi_{2}\left|e_{1}\right|\left|e_{2}\right| & +\chi_{2} e_{2}^{2}-\xi\left|e_{1}\right|-\xi e_{1} u \\
& +\frac{\Gamma(2)}{\Gamma(2-q)} u^{1-q} \operatorname{sign}(u) D^{q} u .
\end{aligned}
$$

Taking advantage on assumption 2, equation becomes:

$$
J \leq \eta e_{1}^{2}-\xi\left|e_{1}\right|-\xi e_{1} u+\frac{\Gamma(2)}{\Gamma(2-q)} u^{1-q} \operatorname{sign}(u) D^{q} u .
$$

If it exists a positive constant $\beta$ such that:

$$
J \leq-\beta \leq 0,
$$

then the update law for the controller $u(t)$ is designed through the following relation:

$$
D^{q} u=\frac{\Gamma(2-q)}{\Gamma(2)} u^{q-1}\left(\xi\left|e_{1}\right|+\xi e_{1} u-\eta e_{1}^{2}-\beta\right) \operatorname{sign}(u) .
$$

For fractional-order $q=1$ the $J$ function becomes the differentiation of the Lyapunov function $(J=\dot{V})$. The theoretical finite-time for synchronization is obtained by integrating equation (15) from 0 to $t_{r}^{1}$. Thus,

$$
\begin{array}{r}
t_{r}^{1} \leq \frac{1}{2 \beta}\left(C_{G S} e_{1}^{2}(0)+C_{G D} e_{2}^{2}(0)+L_{1} e_{3}^{2}(0)+L_{2} e_{4}^{2}(0)\right) \\
+\frac{|u(0)|}{\beta} .
\end{array}
$$

For $0<q<1$, it has been shown in the literature that the fractional controller leads faster to synchronization than the integer controller [22]. It can be concluded that the theoretical finite-time $\left(t_{r}^{q}\right)$ for synchronization of fractional-order simplest two-component oscillators is shorter than the finite-time for synchronization of the same oscillators when $q=1$. Therefore the trajectories of slave fractional-order twocomponent oscillator converge to that of the master oscillator in the finite-time $t_{r}^{q}$, given by $t_{r}^{q} \leq$ $\frac{1}{2 \beta}\left(C_{G S} e_{1}^{2}(0)+C_{G D} e_{2}^{2}(0)+L_{1} e_{3}^{2}(0)+L_{2} e_{4}^{2}(0)\right)+\frac{|u(0)|}{\beta}$. Thus the proof is completed.

\subsection{Numerical simulation}

In this section, the effectiveness and applicability of the proposed finite-time approach is shown. For this simulation, the approximate numerical technique for numerical solving of fractional-order differential equation proposed by Diethelm et al. in [50] is used, which is a generalization of the Adams-Bashforth-Moulton algorithm for ordinary differential equations.

A graphical representation of the synchronization process obtained numerically by carrying out a direct numerical integration on Matlab-Simulink of systems (4), (5) and (8) is provided by Figure 5. The initial conditions for master and slave are respectively: $\left(V_{G S}^{m}, V_{G D}^{m}, i_{1}^{m}, i_{2}^{m}\right)^{T}=$ $(-1.25 \mathrm{~V},-2.5 \mathrm{~V}, 1 \mu \mathrm{A}, 1 \mu \mathrm{A})^{T}$, and $\left(V_{G S}^{s}, V_{G D}^{s}, i_{1}^{s}, i_{2}^{s}\right)^{T}=$ $(0 \mathrm{~V}, 0 \mathrm{~V}, 2 \mu \mathrm{A}, 2 \mu \mathrm{A})^{T}$, with $u(0)=1.6 \eta \mathrm{A}$. To guarantee the existence of chaos in the fractional-order twocomponent oscillator, other parameters are chosen to be $\xi=0.0001, \beta=0.002, E=4 \mathrm{~V}$ and the fractionalorder $q=0.96$. The remaining parameters of systems (4) and (5) are taken as in the first section. Under these conditions the theoretical settled time worth $t_{r T H}^{q}=0.8 \mu \mathrm{s}$. Figure 5a presents the dynamic errors of synchronization between the voltages gate-source and gate-drain of the slave oscillator and their equivalent voltages in the master oscillator, while Figure 5b depicts the dynamic errors 


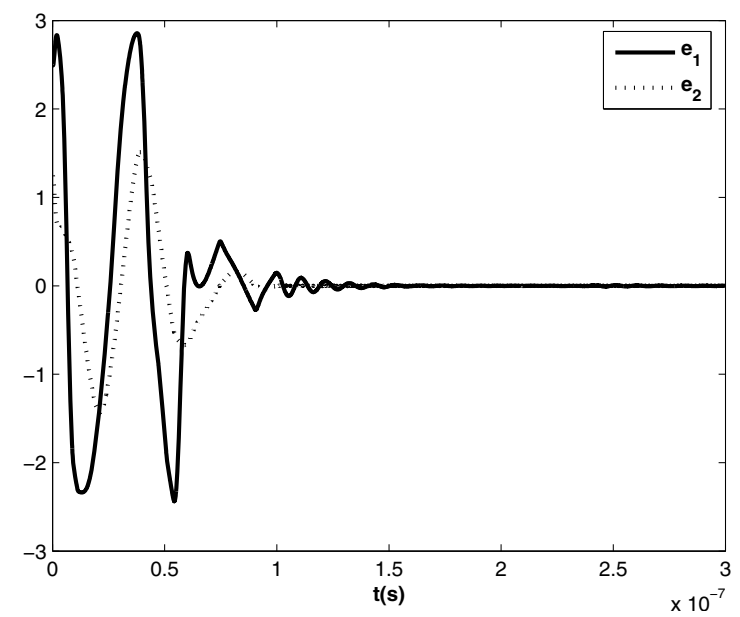

(a)

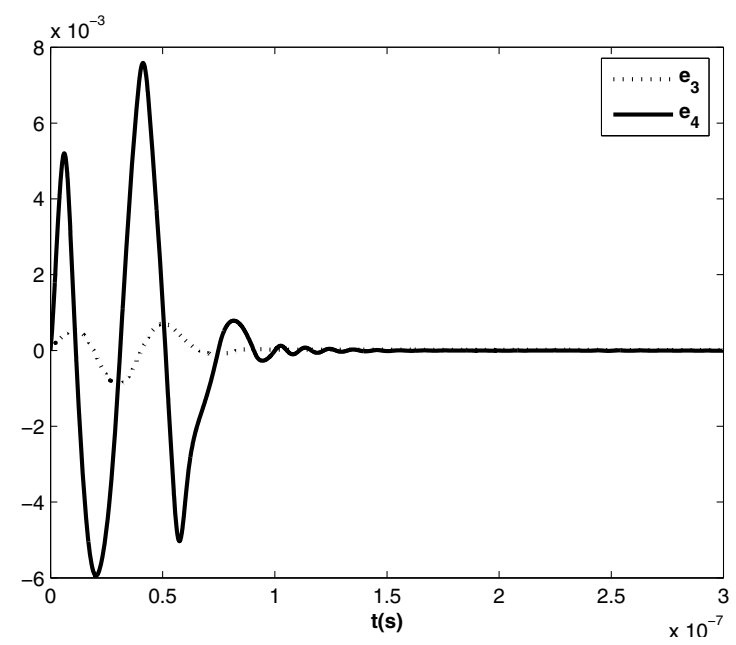

(b)

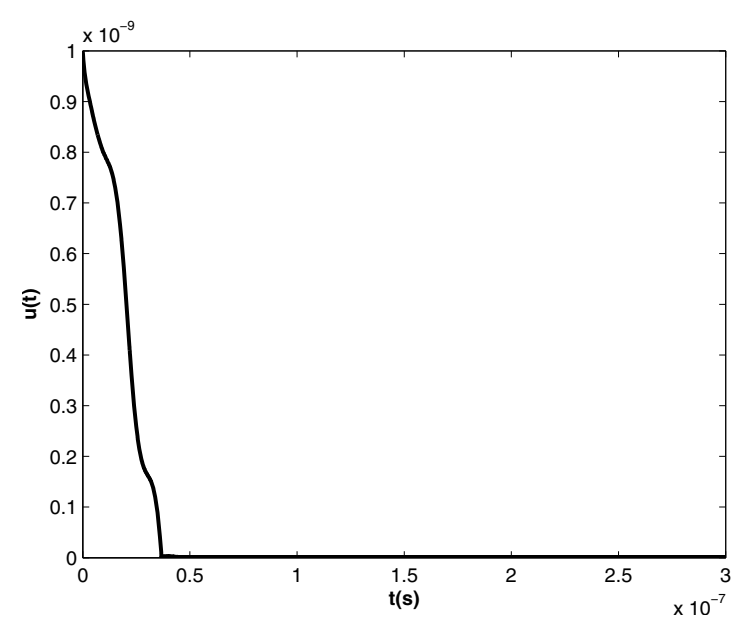

(c)

Fig. 5. Dynamics of synchronization errors (a) on the voltage variables $e_{1}=V_{G S}^{s}-V_{G S}^{m}$ (solid line) and $e_{2}=V_{G D}^{s}-V_{G D}^{m}$ (dashed line) as well as (b) on the current variables $e_{3}=i_{1}^{s}-i_{1}^{m}$ (solid line) and $e_{4}=i_{2}^{s}-i_{2}^{m}$ (dashed line). Graph (c) depicts the time history of the controller $u(t)$, where the numerical finite-time for synchronization is $t_{r N U}^{q}=0.2 \mu \mathrm{s}$. All the curves are simulated for $q=0.96$ and $E=4 \mathrm{~V}$.

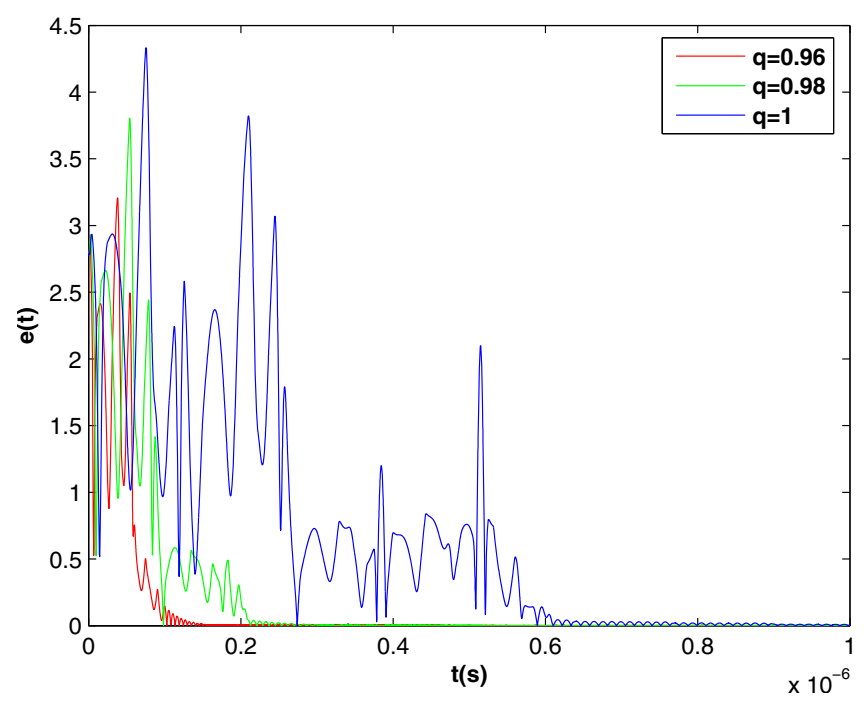

Fig. 6. Time evolution of synchronization errors $e(t)=$ $\sqrt{e_{1}^{2}+e_{2}^{2}+e_{3}^{2}+e_{4}^{2}}$ for different values of fractional-order $q=1$ (blue), $q=0.98$ (green), and $q=0.96$ (red) when the bias voltage source is kept at $E=4 \mathrm{~V}$. It can be noted that the numerical finite-time of synchronization decreases with the fractional-order.

of synchronization between the current flowing through the inductor parts $L_{1}$ and $L_{2}$ of the slave oscillator and the corresponding current in the master one. These graphs show that the numerical finite-time of synchronization is $t_{r N U}^{q}=0.2 \mu \mathrm{s}$, which respects the finite-time condition $t_{r N U} \leq t_{r T H}$ [47]. Figure 5c gives the time evolution of the adaptive feedback controller $u(t)$ which exponentially decreases towards a stable state just before the numerical finite-time synchronization $t_{r N U}$ is reached.

The time evolution of the dynamical synchronization errors $e(t)=\sqrt{e_{1}^{2}+e_{2}^{2}+e_{3}^{2}+e_{4}^{2}}$ is represented (Fig. 6) for different values of the fractional-order $q$, distinguishable by different colors: blue $(q=1)$, green $(q=0.98)$, and red $(q=0.96)$. It can be noted that the numerical finitetime of synchronization decreases with the fractional-order $q$, which confirms the theoretical result as well as the result given by the literature, where it is mentioned that the synchronization of fractional-order chaotic systems is faster than that of integer order chaotic systems [22].

\section{Application to digital cryptography}

\subsection{Algorithm description}

Consider the master oscillator (4) as sender and the slave oscillator (5) as receiver. The laps time between sending and receiving the message must be greater than $t_{r T H}^{q}$. Therefore, after the laps time $t_{r T H}^{q}$, sender and receiver must choose the values of the variable $V_{G S}^{m}$ respectively $V_{G S}^{s}$, to get the secret keys. The elements of the Fibonacci $Q$ matrix are used to generate the needed secret keys. It can be referred to references [51-55] for more details on the theory of Fibonacci numbers. The $n$th power of the $Q$ 


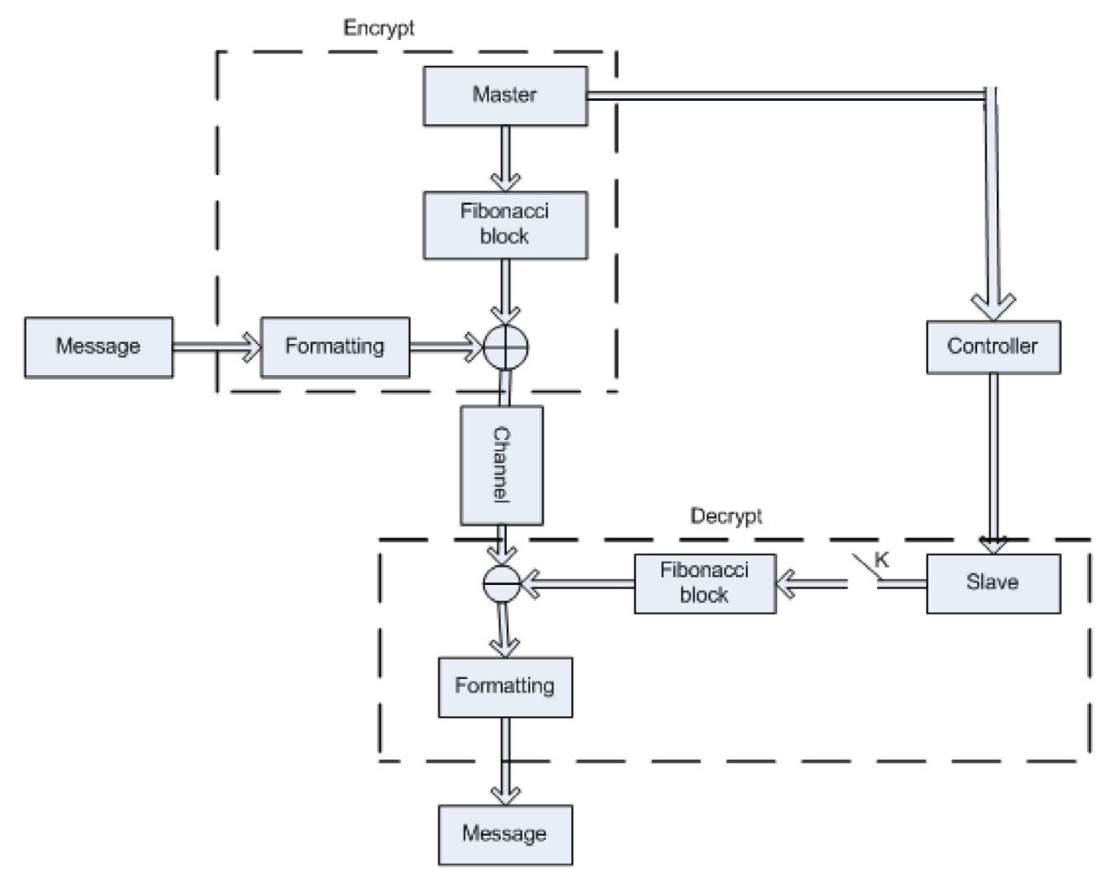

Fig. 7. Schematics of the master and slave control systems.

matrix is defined as:

$$
Q^{n}=\left(\begin{array}{cc}
F_{n+1} & F_{n} \\
F_{n} & F_{n-1}
\end{array}\right) .
$$

Here the Fibonacci numbers $F_{n}, n \geq 3$ is defined by $F_{n+1}=F_{n}+F_{n-1}$, taking into account that the initials terms are $F_{0}=0$ and $F_{1}=F_{2}=1$.

The plaintext $(p)$ and the corresponding ciphertext (c) can be divided into message sequences or units of $2 \times 2$ square matrixes. The assignment of numbers to message units respects the ASCII code table. The ciphertext and decrypted message corresponding to the assignment of numbers can be obtained according to the following system:

$$
\left\{\begin{array}{l}
c=p+k(\bmod 128) \\
p=c-k(\bmod 128)
\end{array}\right.
$$

The secret keys for the sender and receiver respectively can now be written as $k_{s}=\left[F \cdot t \cdot\left|V_{G S}^{m}\right| \cdot q\right](\bmod 128)$ and $k_{r}=\left[F \cdot t \cdot\left|V_{G S}^{s}\right| \cdot q\right](\bmod 128) . F$ stands for the frequency of the fractional chaotic oscillator, meanwhile the term in bracket, say $[\varrho]$ denotes the integer part of $\varrho$, and $|\sigma|$ would correspond to the modulus value of the variable $\sigma$. The rest of the algorithm remains the same as defined in reference [22]. Note that in contrary to [22], we performed finite-time synchronization which is more appropriate for digital cryptography. The master oscillator slave oscillator configuration is presented in Figure 7.

\subsection{Numerical example}

Consider the test message "2 YEARS OLD" that has to be transmitted. It has eleven message units including blanks.
To set $2 \times 2$ square matrixes constituting the plaintext blocks, it is necessary that the test message length be a multiple of four. This can be achieved if one supplementary blank space is inserted at the end of the above test message which length will become twelve and therefore, a multiple of four. Consequently, sequences of message units will be put in $2 \times 2$ square matrix blocks, say $M_{1}$ for the four first plaintext, $M_{2}$ for the second four and $M_{3}$ for the last. The transcription of those matrixes using ASCII code becomes:

$$
M_{1}=\left(\begin{array}{ll}
50 & 32 \\
89 & 69
\end{array}\right), M_{2}=\left(\begin{array}{ll}
65 & 82 \\
83 & 32
\end{array}\right), M_{3}=\left(\begin{array}{ll}
79 & 76 \\
68 & 32
\end{array}\right) .
$$

At $t_{1}=1 \mu s\left(t_{1}>t_{r T H}^{q}\right)$, corresponding to $V_{G S}^{m}=$ $0.2264 \mathrm{~V}$, a secret key $k_{s}=\left[F \cdot t \cdot\left|V_{G S}^{m}\right| \cdot q\right](\bmod 128)=$ $\left[150 \times 10^{6} \times 10^{-6} \times(0.2264) \times(0.96)\right]=32(\bmod 128)$ for the sender is built and the first block of message unit $M_{1}$ is sent. The Fibonacci number corresponding to that first

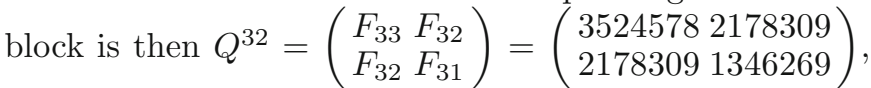
hence the first four keys are: $k_{1 s}=F_{33}(\bmod 128)=98$, $k_{2 s}=k_{3 s}=F_{32}(\bmod 128)=5, k_{4 s}=F_{31}(\bmod 128)=93$. It follows the ciphertext below

$$
\left(\begin{array}{ll}
c_{1} & c_{2} \\
c_{3} & c_{4}
\end{array}\right)=\left(\begin{array}{ll}
p_{1} & p_{2} \\
p_{3} & p_{4}
\end{array}\right)+\left(\begin{array}{ll}
k_{1 s} & k_{2 s} \\
k_{3 s} & k_{4 s}
\end{array}\right)=\left(\begin{array}{cc}
148 & 37 \\
94 & 162
\end{array}\right) .
$$

At the reception end the secret key for the decoding of the test message is $k_{r}=\left[F \cdot t \cdot\left|V_{G S}^{s}\right| \cdot q\right](\bmod 128)=[150$. $\left.10^{6} \times 10^{-6} \times(0.2264) \times(0.96)\right](\bmod 128)=32(\bmod 128)$. The receiver can decode the message as it follows, using equation (19):

$$
\left(\begin{array}{ll}
p_{1} & p_{2} \\
p_{3} & p_{4}
\end{array}\right)=\left(\begin{array}{ll}
c_{1} & c_{2} \\
c_{3} & c_{4}
\end{array}\right)-\left(\begin{array}{ll}
k_{1 r} & k_{2 r} \\
k_{3 r} & k_{4 r}
\end{array}\right)=\left(\begin{array}{ll}
50 & 32 \\
89 & 69
\end{array}\right) .
$$


Table 1. Details of sending and receiving a text message.

\begin{tabular}{cccccccc}
\hline Time $t$ & $\left|V_{G S}^{m}\right|$ & Plaintext $p$ & Keys $k_{s}$ & Ciphertext $c$ & $\left|V_{G S}^{s}\right|$ & Keys $k_{r}$ & Plaintext recovered \\
\hline $1 \mu \mathrm{s}$ & $0.2264 \mathrm{~V}$ & $2(50)$ & $k_{1 s}=98$ & 149 & $0.2264 \mathrm{~V}$ & $k_{1 r}=98$ & $50(2)$ \\
& & $-(32)$ & $k_{2 s}=5$ & 37 & & $k_{2 r}=5$ & $32(-)$ \\
& & $\mathrm{Y}(89)$ & $k_{3 s}=5$ & 94 & & $k_{3 r}=5$ & $89(\mathrm{Y})$ \\
$2 \mu \mathrm{s}$ & \multirow{3}{*}{$0.57 \mathrm{~V}$} & $\mathrm{E}(69)$ & $k_{4 s}=93$ & 162 & & $k_{4 r}=93$ & $69(\mathrm{E})$ \\
& & $\mathrm{A}(65)$ & $k_{1 s}=121$ & 186 & $0.57 \mathrm{~V}$ & $k_{1 r}=121$ & $65(\mathrm{~A})$ \\
& & $\mathrm{R}(82)$ & $k_{2 s}=48$ & 130 & & $k_{2 r}=48$ & $82(\mathrm{R})$ \\
$3 \mu \mathrm{S}$ & $\mathrm{S}(83)$ & $k_{3 s}=48$ & 131 & & $k_{3 r}=48$ & $83(\mathrm{~S})$ \\
& \multirow{3}{*}{$0.1738 \mathrm{~V}$} & $-(32)$ & $k_{4 s}=73$ & 105 & & $k_{4 r}=73$ & $32(-)$ \\
& & $\mathrm{O}(79)$ & $k_{1 s}=83$ & 162 & $0.1738 \mathrm{~V}$ & $k_{1 r}=83$ & $79(\mathrm{O})$ \\
& & $\mathrm{L}(76)$ & $k_{2 s}=66$ & 142 & & $k_{2 r}=66$ & $76(\mathrm{~L})$ \\
& & $\mathrm{D}(68)$ & $k_{3 s}=66$ & 134 & & $k_{3 r}=66$ & $68(\mathrm{D})$ \\
& & $-(32)$ & $k_{4 s}=17$ & 49 & & $k_{4 r}=17$ & $32(-)$ \\
\hline
\end{tabular}

Table 2. Effect of time $t$ on the digital cryptography for $q=0.96$.

\begin{tabular}{cccccccc}
\hline Time $t$ & $\left|V_{G S}^{m}\right|$ & Plaintext $p$ & Keys $k_{s}$ & Ciphertext $c$ & $\left|V_{G S}^{s}\right|$ & Keys $k_{r}$ & Plaintext recovered \\
\hline $0.038 \mu \mathrm{s}$ & $0.91 \mathrm{~V}$ & $2(50)$ & $k_{1 s}=5$ & 55 & $0.4716 \mathrm{~V}$ & $k_{1 r}=2$ & $53(5)$ \\
& & $-(32)$ & $k_{2 s}=3$ & 35 & & $k_{2 r}=1$ & $34(")$ \\
& & $\mathrm{Y}(89)$ & $k_{3 s}=3$ & 92 & & $k_{3 r}=1$ & $91([)$ \\
& $\mathrm{E}(69)$ & $k_{4 s}=2$ & 71 & & $k_{4 r}=1$ & $70(\mathrm{~F})$ \\
\hline $0.2 \mu \mathrm{s}$ & $0.4627 \mathrm{~V}$ & $2(50)$ & $k_{1 s}=121$ & 171 & $0.4627 \mathrm{~V}$ & $k_{1 r}=121$ & $50(2)$ \\
& & $-(32)$ & $k_{2 s}=105$ & 137 & & $k_{2 r}=105$ & $32(-)$ \\
& $\mathrm{Y}(89)$ & $k_{3 s}=105$ & 194 & & $k_{3 r}=105$ & $89(\mathrm{Y})$ \\
& $\mathrm{E}(69)$ & $k_{4 s}=16$ & 85 & & $k_{4 r}=16$ & $69(\mathrm{E})$ \\
\hline
\end{tabular}

The complete procedure of sending and recovering the message is summarized in Table 1.

\subsection{Effect of the variable time on cryptography}

The variables $V_{G S}^{m}$ and $V_{G S}^{s}$ proceed from the time dependent fractional-order chaotic systems operating at high frequency. Table 2 shows the effect of the variable $t$ on cryptography when the fractional-order is fixed at $q=$ 0.96. In this table we experienced two values of time: for $t_{1}=0.038 \mu \mathrm{s}$, less than the finite-time of synchronization (see Fig. 5), we observed that the synchronization between the drive and the response systems is not established, because a non-zero difference between $V_{G S}^{m}$ and $V_{G S}^{s}$ do exist. This difference between the states of the master and those of the slave leads to a difference between the sending keys and the receive keys. The consequence is direct: the recovered message is different from the transmitted one. On the other hand when $t_{1}=0.2 \mu \mathrm{s}$ is chosen greater than the finite-time of synchronization, the states of master $V_{G S}^{m}$ and slave $V_{G S}^{s}$ are quite identical. Consequently, the received keys coincide now with those of sending and the plaintext recovered on arrival is the same as the sent plaintext $p$. These results show the importance of control at finite-time of synchronization and confirm the effectiveness of our model.

\subsection{Effect of fractional-order on cryptography}

Here we study the effect of fractional-order $q$ on the digital cryptography when the time value is fixed at $t_{1}=0.2 \mu \mathrm{s}$.
Table 3 shows that the plaintext is recovered solely in the case where the fractional-order worths $q=0.96$, because this is the only case in which the states both of the master $V_{G S}^{m}$ and of the slave $V_{G S}^{s}$ are identical. This result can be explained by the fact that for $q=0.98$ and $q=1$ the time $t_{1}=0.2 \mu \mathrm{s}$ is less than the finite-time of synchronization (Fig. 5).

\section{Conclusions}

In this paper, the problem of finite-time synchronization of fractional-order simplest two-component chaotic oscillator operating at high frequency and application to digital cryptography was studied. An efficient finite-time adaptive controller was introduced, on the basis of fractional Lyapunov stability theory. An estimation of the convergence time was also given. Numerical simulation were performed and given to confirm our theoretical analysis. Furthermore, it was shown that the synchronization of fractional-order systems is faster than that of integer ones. Finally, a proposed key systems for cryptographic encryption scheme with the help of the finite-time synchronization of fractional-order systems was illustrated through a numerical example, showing that a high level security can be produced.

From the security analysis of the proposed key system, we conclude that the proposed key system in the present paper is an improvement of the key system proposed in reference [22]. Indeed through the secret elements $V_{G S}^{m}$, $V_{G S}^{s}, t$, and $q$ we also control the time of synchronization which is an additional advantage as presented in Section 4. 
Table 3. Effect of fractional-order $q$ on the digital cryptography for $t=0.2 \mu$.

\begin{tabular}{cccccccc}
\hline$q$ & $\left|V_{G S}^{m}\right|$ & Plaintext $p$ & Keys $k_{s}$ & Ciphertext $c$ & $\left|V_{G S}^{s}\right|$ & Keys $k_{r}$ & Plaintext recovered \\
\hline 0.96 & $0.4627 \mathrm{~V}$ & $2(50)$ & $k_{1 s}=121$ & 171 & $0.4627 \mathrm{~V}$ & $k_{1 r}=121$ & $50(2)$ \\
& & $-(32)$ & $k_{2 s}=105$ & 137 & & $k_{2 r}=105$ & $32(-)$ \\
& & $\mathrm{Y}(89)$ & $k_{3 s}=105$ & 194 & & $k_{3 r}=105$ & $89(\mathrm{Y})$ \\
& & $\mathrm{E}(69)$ & $k_{4 s}=16$ & 85 & & $k_{4 r}=16$ & $69(\mathrm{E})$ \\
\hline 0.98 & $0.512 \mathrm{~V}$ & $2(50)$ & $k_{1 s}=85$ & 135 & $0.613 \mathrm{~V}$ & $k_{1 r}=91$ & $44()$, \\
& & $-(32)$ & $k_{2 s}=24$ & 56 & & $k_{2 r}=98$ & -42() \\
& & $\mathrm{Y}(89)$ & $k_{3 s}=24$ & 113 & & $k_{3 r}=98$ & $15(\mathrm{C}-\mathrm{o})$ \\
& $\mathrm{E}(69)$ & $k_{4 s}=61$ & 130 & & $k_{4 r}=121$ & $7(\mathrm{C}-\mathrm{g})$ \\
\hline 1 & $1.295 \mathrm{~V}$ & $2(50)$ & $k_{1 s}=85$ & 135 & $0.61 \mathrm{~V}$ & $k_{1 r}=34$ & $101(\mathrm{e})$ \\
& $-(32)$ & $k_{2 s}=24$ & 56 & & $k_{2 r}=41$ & $15(\mathrm{C}-\mathrm{o})$ \\
& & $\mathrm{Y}(89)$ & $k_{3 s}=24$ & 113 & & $k_{3 r}=41$ & $89(\mathrm{H})$ \\
& $\mathrm{E}(69)$ & $k_{4 s}=61$ & 130 & & $k_{4 r}=121$ & $69(\mathrm{C}-\mathrm{i})$ \\
\hline
\end{tabular}

It is also worth noticing that the introduced fractional control method can be applied to control a large class of uncertain nonlinear fractional-order dynamical systems in finite time.

G.L. gratefully acknowledges the support of the Polish National Science Center under Grant No. 2012/05/B/ST8/00080.

\section{Author contribution statement}

RK and RT conceived the study; RK, AM and RT did the calculations; RK and AF did computer simulation; RK, RT and GL wrote the manuscript.

Open Access This is an open access article distributed under the terms of the Creative Commons Attribution License (http://creativecommons.org/licenses/by/4.0), which permits unrestricted use, distribution, and reproduction in any medium, provided the original work is properly cited.

\section{References}

1. R. Hilfer, Applications of Fractional Calculus in Physics (World Scientific, Singapore, 2001)

2. S. Müller, M. Kästner, J. Brummund, V. Ulbricht, Comput. Mater. Sci. 50, 2938 (2011)

3. Y. Luo, Y.Q. Chen, Y. Pi, Mechatronics 21, 204 (2011)

4. N. Laskin, Physica A 287, 482 (2000)

5. M. Rivero, J.J. Trujillo, L. Vazquez, M.P. Velasco, Appl. Math. Comput. 218, 1089 (2011)

6. N. Ozalp, E. Demirci, Math. Comput. Model 54, 1 (2011)

7. J. Cao, C. Ma, Z. Jiang, S. Liu, Commun. Nonlinear Sci. Numer. Simul. 16, 1443 (2011)

8. C. Ionescu, J.T. Machado, R. de Keyser, Comput. Math. Appl. 62, 845 (2011)

9. J. Cao, A. Syta, G. Litak, S. Zhou, D.J. Inman, Y. Chen, Eur. Phys. J. Plus 130, 103 (2015)

10. C.A.K. Kwuimy, G. Litak, C. Nataraj, Nonlinear Dynamics 80, 491 (2015)

11. B. Ducharne, G. Sebald, D. Guyomar, G. Litak, J. Appl. Phys. 117, 243907 (2015)
12. I. Grigorenko, E. Grigorenko, Phys. Rev. Lett. 91, 034101 (2003)

13. T.T. Hartley, C.F. Lorenzo, H.K. Qammer, IEEE Trans. Circuits Syst. 42, 485 (1995)

14. Z.M. Ge, A.R. Zhang, Chaos Solitons Fractals 32, 1791 (2007)

15. Z.M. Ge, C.Y. Ou, Chaos Solitons and Fractals 35, 705 (2008)

16. J.G. Lu, Chaos Solitons and Fractals 26, 1125 (2005)

17. D.G. Varsha, B. Sachin, J. Comput. Appl. Math. 59, 1117 (2010)

18. C. Zhang, S. Yu, Chaos Solitons and Fractals 44, 845 (2011)

19. R.-H. Li, W.-S. Chen, Chin. Phys. B 22, 040503 (2013)

20. M.-F. Danca, Nonlinear Dyn. 60, 525 (2010)

21. D. Cafagna, G. Grassi, Nonlinear Dyn. 70, 1185 (2012)

22. P. Muthukumar, P. Balasubramaniam, Nonlinear Dyn. 74, 1169 (2013)

23. R. Kengne, R. Tchitnga, A. Tchagna Kouanou, A. Fomethe, J. Engineering Sci. Technology Rev. 6, 24 (2013)

24. S. Hayes, C. Grebogi, E. Ott, Phys. Rev. Lett. 70, 3031 (1993)

25. L. Kocarev, U. Parlitz, Phys. Rev. Lett. 74, 5028 (1995)

26. D. Chen, C. Liu, C. Wu, Y. Liu, X. Ma, Y. You, Circuits Syst. Signal Process 31, 1599 (2012)

27. H. Li, X. Liao, M. Luo, Nonlinear Dyn. 68, 137 (2012)

28. B. Wang, J. Xue, D. Chen, Journal of vibration and Control, DOI : $10.1177 / 1077546314546682$

29. C.-M. Chang, H.-K. Chen, Nonlinear Dyn. 62, 851 (2010)

30. C. Li, Y. Tong, Pramana J. Phys. 80, 583 (2013)

31. Y. Tang, J.-A. Fang, Commun. Nonlinear Sci. Numer. Simulat. 15, 401 (2010)

32. J.G. Lu, Phys. Lett. A 354, 305 (2006)

33. P. Zhou, R. Ding, J. Franklin Inst. 348, 2839 (2011)

34. G.J. Peng, Y.L. Jiang, Phys. Lett. A 372, 3963 (2008)

35. C.G. Li, X. Liao, J.B. Yu, Phys. Rev. E 68, 067203 (2003)

36. P. Louodop, H. Fotsin, M. Kountchou, S. Bowong, Phys. Scr. 88, 045002 (2013)

37. P. Louodop, M. Kountchou, H. Fotsin, S. Bowong, Nonlinear Dyn. 78, 597 (2014)

38. M.P. Aghababa, J. Comput. Nonlinear Dyn. 7, 021010 (2012)

39. R. Kengne, R. Tchitnga, A. Tchagna, T.A. Kouanou, Nzeusseu, A. Fomethe, J. Chaos 2013, 839038 (2013)

40. M.P. Aghababa, Nonlinear Dyn. 69, 247 (2012)

41. B. Nana, S.B. Yamgoué, R. Tchitnga, P. Woafo, Am. J. Electrical and Electronic Engineering 3, 29 (2015) 
42. R. Tchitnga, R. Zebaze Nanfaa, F.B. Pelap, P. Louodop, P. Woafo, J. Vibration and Control, DOI : $10.1177 / 1077546315585424$

43. R. Tchitnga, H.B. Fotsin, B. Nana, P. Louodop, P. Woafo, Chaos Solitons and Fractals 45, 306 (2012)

44. G.F. Joana, J.A.C. Gallas, Chaos Solitons and Fractals 59, $129(2014)$

45. R.M. Nguimdo, R. Tchitnga, P. Woafo, Chaos 23, 043122 (2013)

46. C. Li, J. Zhang, Int. J. Syst. Sci. 47, 2440 (2016)

47. P. Louodop, H. Fotsin, M. Kountchou, H.A. Cerdeira, E.B. Megam Ngouonkadi, S. Bowong, Phys. Rev. E 89, 032921 (2014)
48. A. Wolf, J.B. Swift, H.L. Swinney, J.A. Vastano, Physica D 16, $285(1985)$

49. A. Syta, G. Litak, S. Lenci, M. Scheffler, Chaos 24, 013107 (2015)

50. K. Diethelm, N.J. Ford, A.D. Freed, Nonlinear Dyn. 13, 3 (2002)

51. M.S. El Naschie, Comput. Math. Appl. 29, 103 (1995)

52. V.E. El Hoggat, Fibonacci and Lucas Numbers (Houghton Mifflin, Palo Alto, 1969)

53. H.W. Gould, Fibonacci Q 19, 250 (1981)

54. J.R. Silvester, Math. Gaz. 63, 188 (1979)

55. O.P. Stakhov, Rep. Natl. Acad. Sci. 9, 46 (1999) 